\title{
Baseline Plasma EGFR Circulating Tumour DNA Levels in a Pilot Cohort of EGFR-Mutant Limited-Stage Lung Adenocarcinoma Patients Undergoing Radical Lung Radiotherapy
}

\author{
Brendan Seng Hup Chia ${ }^{a}$ Wen Long Nei ${ }^{a}$ Sabanayagam Charumathi ${ }^{b}$ \\ Kam Weng Fong a Min-Han Tan ${ }^{c}$ \\ aDivision of Radiation Oncology, National Cancer Centre Singapore, Singapore, Singapore; \\ ${ }^{b}$ Centre for Quantitative Medicine, Duke-NUS Medical School, Singapore, Singapore; \\ 'Institute of Bioengineering and Nanotechnology, A*STAR Singapore, Singapore, Singapore
}

\section{Keywords}

Lung adenocarcinoma · Plasma EGFR · Circulating tumour DNA - Biomarker · Prognosis

\begin{abstract}
The use of circulating cell-free tumour DNA (ctDNA) is established in metastatic lung adenocarcinoma to detect and monitor sensitising EGFR mutations. In early-stage disease, there is very little data supporting its role as a potential biomarker. We report on a prospective cohort of 9 limited-stage EGFR mutant lung cancer patients who were treated with radical radiotherapy. We looked at baseline plasma EGFR ctDNA and noted the detection rates to be higher in locally advanced disease. At a median follow-up of 13.5 months, an association between a detectable pre-radiotherapy plasma EGFR ctDNA and early tumour relapse ( 155 days vs. NR, $p=0.004$ ) was noted. One patient with persistent plasma EGFR ctDNA predated radiological progression. The role of ctDNA in early-stage lung cancer is developing. Plasma EGFR ctDNA could be a useful biomarker in lung cancer patients undergoing radical treatments for staging, prognostication, and follow-up. These preliminary findings should be explored in larger studies.

(c) 2020 The Author(s).

Published by S. Karger AG, Basel
\end{abstract}

\section{Introduction}

Lung cancer is one of the commonest cancers worldwide and the leading cause of cancer deaths. The presence of targetable epidermal growth factor receptor (EGFR) mutations in the tumour has allowed for the use of tailored treatments like tyrosine kinase inhibitors (TKIs) 
to significantly prolong survival in some patients. In East Asia, the prevalence of these mutations is around $38.4 \%$ in non-small cell lung carcinoma (NSCLC) [1].

Circulating tumour DNA (ctDNA) are tumour-specific DNA fragments that are shed into the blood stream. Its presence provides an opportunity to use non-invasive blood biomarkers to detect mutations, including EGFR. Multiplex PCR or next-generation sequencing (NGS) based platforms are available to detect ctDNA. Recently, this technology has been approved by regulators for use in diagnosis, guiding treatment, and monitoring emergence of resistant mutations in metastatic NSCLC $[2,3]$. Some groups have also proposed monitoring the plasma EGFR ctDNA levels for dynamic changes to predict treatment failure of TKIs [4-6].

In stage I-III NSCLC, the use of ctDNA in these group of patients is still experimental. In surgical series, it has been suggested that ctDNA could be used to detect minimal residual disease and predict early recurrences [7-10]. There is limited evidence that ctDNA could be used to monitor patients who have undergone radiotherapy $[8,11]$.

After radical lung radiotherapy, a portion of patients may suffer high-grade treatmentrelated toxicities and a significant proportion will still relapse despite this. The interpretation of radiological changes after radiotherapy is also complicated by infective or inflammatory changes, scarring and fibrosis resulting in delayed diagnosis. There is thus an unmet need for a non-invasive biomarker that could allow clinicians to prognosticate and predict for treatment failures in these patients.

Our primary aim of this study was to evaluate the use of baseline plasma EGFR ctDNA in a cohort of EGFR mutant patients undergoing radical lung radiotherapy. We hypothesised that patients with a detectable pre-treatment plasma EGFR ctDNA would reach a higher disease stage, which may correlate with a poorer survival outcome than in those without.

\section{Case Presentation}

\section{Patients}

We conducted a pilot prospective cohort study from April 2017 to August 2018 in the National Cancer Centre Singapore for patients referred to the radiotherapy department for radical lung radiotherapy. The protocol and consent forms were reviewed and approved by SingHealth Institution Review Board. All patients were required to sign informed consent prior to enrolment. The participants had to be fully staged with PET/CT, MRI brain, and EBUS before recruitment. Only radically treatable stage I-III lung adenocarcinoma with a biopsy-proven targetable EGFR mutation in exons 18-21 [11] were recruited. Patients were recruited consecutively. Prior use of chemotherapy was not an exclusion criteria.

\section{Plasma EGFR Mutation ctDNA Procedure}

Twenty millilitres of blood (4 EDTA tubes) were collected at CT-simulation scans, prior to radiotherapy, for measurement of baseline test. The blood collected was processed within $2 \mathrm{~h}$ and stored for ctDNA extraction. Detection of plasma EGFR mutations, including p.E746_ A750del, p.L858R, and p.T790M, was conducted by amplification refractory mutation system (ARMS) PCR. Laboratory staff were blinded to patient and tumour details to avoid read-out bias of PCR results. A second test was planned 1 month after radiotherapy. The results of the tests were blinded to the treating physician and patient.

The protocol was later amended to allow for use of multi gene panel (gene list: $A L K, B R A F$, ERBB2, EGFR [exons 18-21], KRAS, MET, NRAS, PIK3CA, STK11, and TP53) NGS LiquidMARK ${ }^{\mathrm{TM}}$ test [12] on the stored frozen plasma samples.

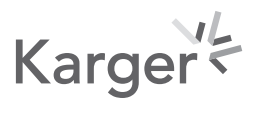




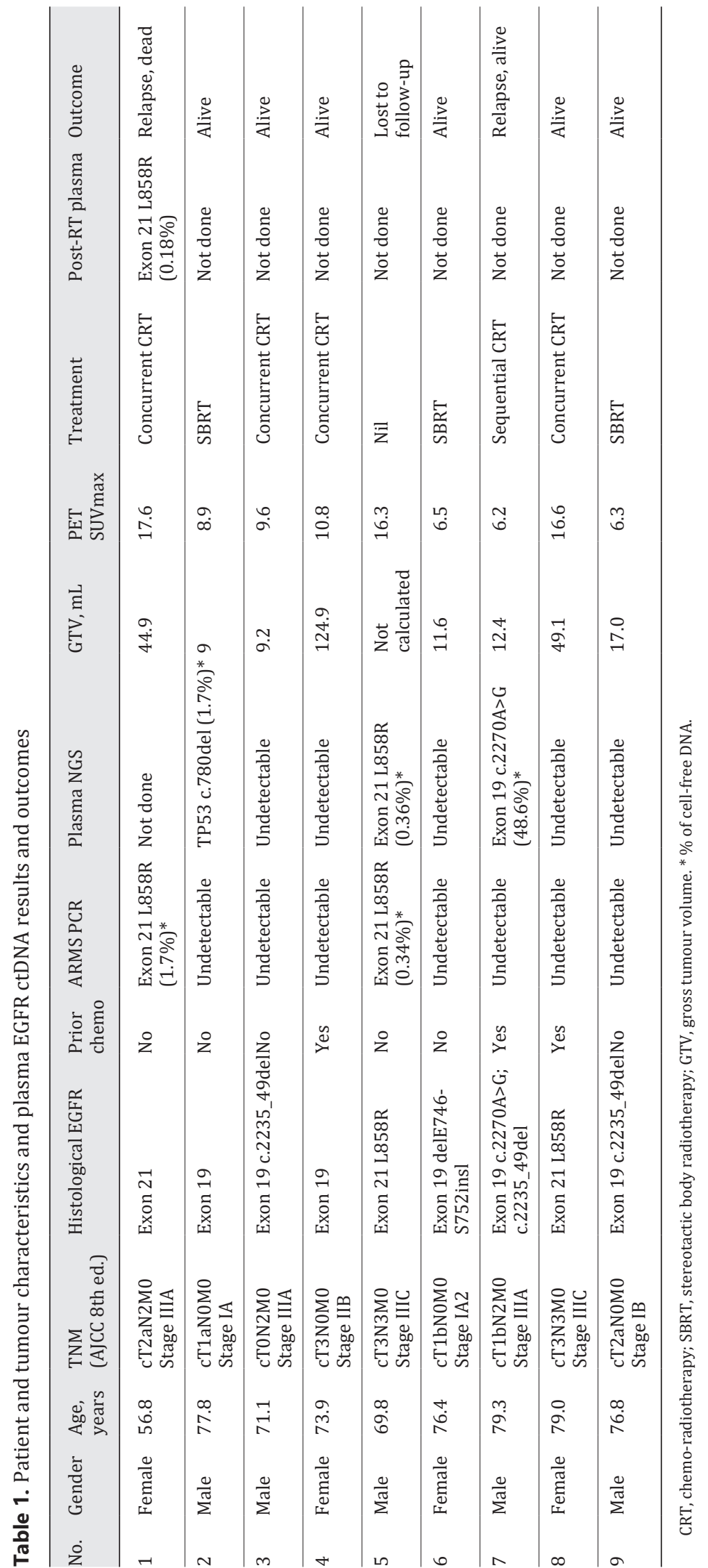




\section{Case Reports in Oncology}

Table 2. Test for factors on detection of plasma EGFR ctDNA

\begin{tabular}{l|l}
\hline Case Rep Oncol 2020;13:896-903 \\
\hline DOI: 10.1159/000508932 & $\begin{array}{l}\text { @ 2020 The Author(s). Published by S. Karger AG, Basel } \\
\text { www.karger.com/cro }\end{array}$ \\
\hline
\end{tabular}

\begin{tabular}{|c|c|c|c|}
\hline \multirow[t]{2}{*}{ Variable } & \multicolumn{2}{|c|}{ Plasma EGFR ctDNA } & \multirow[t]{2}{*}{$p$ value } \\
\hline & Detected & Undetected & \\
\hline Gender & & & $1.00 *$ \\
\hline Male $(n=5)$ & 2 & 3 & \\
\hline Female $(n=4)$ & 1 & 3 & \\
\hline Stage & & & $0.167^{*}$ \\
\hline III $(n=5)$ & 3 & 2 & \\
\hline I/II $(n=4)$ & 0 & 4 & \\
\hline Pre-ARMS PCR chemotherapy & & & $0.774^{*}$ \\
\hline Yes $(n=3)$ & 1 & 2 & \\
\hline No $(n=6)$ & 2 & 4 & \\
\hline Mean GTV, mL & 28.7 & 36.8 & $0.759^{\wedge}$ \\
\hline Mean SUVmax & 13.4 & 9.8 & $0.433^{\wedge}$ \\
\hline
\end{tabular}

* Fisher's exact test. ${ }^{\wedge}$ Welch $t$ test.

\section{Baseline and Follow-Up Evaluation}

The patient's characteristics, tumour details and, treatment plans were recorded at baseline, and treatment outcomes (local or distant relapses) were recorded at the 3-4 monthly follow-up. Survival outcomes were recorded from time of blood taking to event or last followup visit. Patients lost to follow-up would be censored at last visit. Patient who did not complete the radical treatment would not be assessed for outcome studies.

\section{Statistics}

Baseline characteristics of patients were compared with plasma EGFR ctDNA detection rate, these were also compared against relapse/death outcomes. We used $\chi^{2}$ test for categorical data and the $t$ test for continuous data. Time to event was taken from the start of radiotherapy. Kaplan-Meier method was used to detect the differences between survival outcomes. The impact of plasma EGFR ctDNA test results on survival outcomes was evaluated by log-rank test. $p$ values are two-tailed and those below 0.05 were considered statistically significant. Statistical analysis was conducted using IBM SPSS statistics ver. 25.

\section{Results}

\section{Patient Characteristics}

During the trial enrolment period, 9 patients consented to the study. Patient and tumour characteristics are summarised in Table 1 . One patient subsequently declined radiotherapy and was lost to follow-up. This patient was excluded from outcome analysis. Eight underwent radical lung radiotherapy (3 SBRT, 5 chemo-radiotherapy).

\section{Plasma EGFR ctDNA Test Results}

Two of $9(22.2 \%)$ participants had detectable results at baseline using ARMS PCR. In combining this with NGS, 4 of $9(44.4 \%)$ participants had detectable ctDNA (3 EGFR and 1 TP53 mutations) at baseline. The 3 patients who had a detectable baseline plasma EGFR ctDNA had stage IIIA/C disease at diagnosis. None of the 4 stage I/II patients had a detectable plasma EGFR ctDNA result. The sensitivity of tests in detecting plasma EGFR ctDNA in stage III was $60 \%$ versus $0 \%$ in stage I/II patients, although this result was not statistically significant. No patient and tumour variable studied had a significant correlation with baseline plasma EGFR ctDNA detection (Table 2). Only 1 patient did a 1-month post-treatment test.

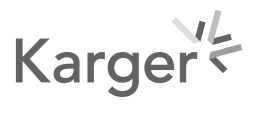




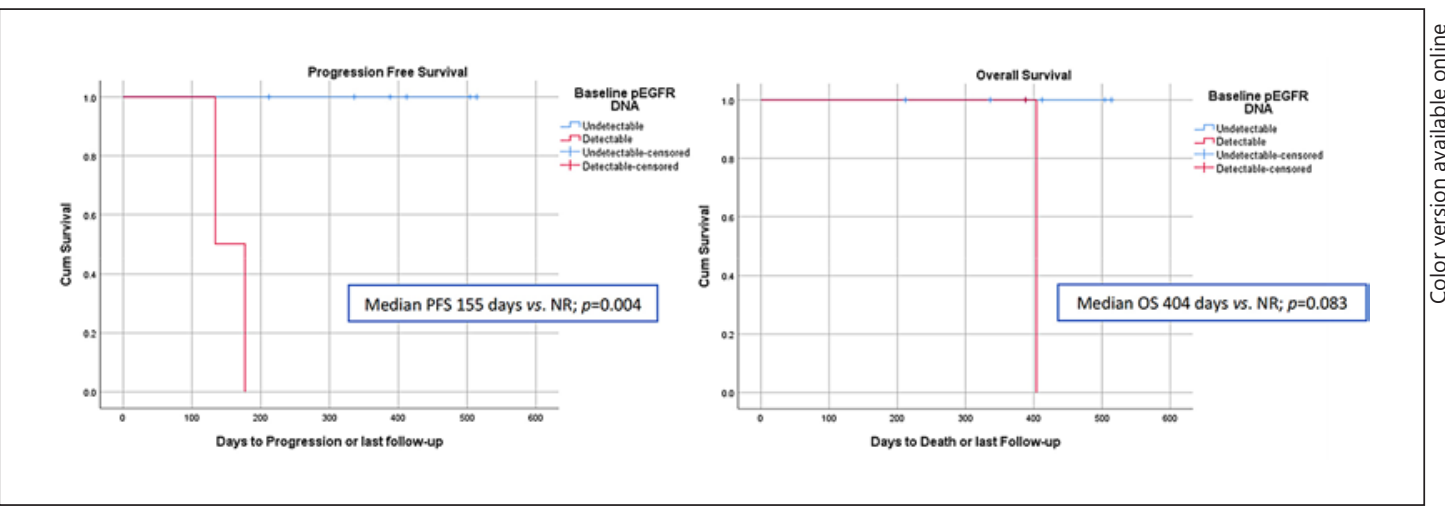

Fig. 1. Progression-free survival and overall survival curves stratified by plasma EGFR ctDNA detection.

Table 3. Characteristics of participants by outcome (relapse/death)

\begin{tabular}{llll}
\hline Characteristics & \multicolumn{2}{l}{ Outcome } & \multirow{2}{*}{$p$ value } \\
\cline { 2 - 3 } & Yes & No & \\
\hline Age, years (SD) & $68.0(15.9)$ & $75.8(2.9)$ & $0.2^{\wedge}$ \\
$\begin{array}{l}\text { Gender, female } \\
\text { Baseline plasma EGFR ctDNA }\end{array}$ & 1 & 3 & $0.6^{*}$ \\
$\quad$ Detected & 2 & 0 & $0.01^{*}$ \\
$\quad$ Undetected & 0 & 6 & \\
Stage & 2 & 2 & $0.1^{*}$ \\
$\quad$ III & 0 & 4 & \\
$\quad$ I/II & 0 & & \\
\hline
\end{tabular}

${ }^{*}$ Fisher's exact test. ${ }^{\wedge}$ Welch $t$ test.

\section{Survival Data}

Of the 8 patients who completed radical radiotherapy, the overall median duration follow-up was 396 days (212-514 days). At last follow-up, 6 patients remained disease free and 2 patients had distant metastatic relapse, of which 1 passed away subsequently. The latter had detectable plasma EGFR ctDNA at 1 month post-treatment and had radiological metastatic disease progression 48 days after.

On analysis, patients with a detectable baseline plasma EGFR ctDNA detection were associated with relapse/death outcome $(p=0.01)$ (Table 3). These patients also had a shorter median PFS (155 days vs. NR, $p=0.004$ ), although OS was not significantly different (404 days vs. NR; $p=0.083$ ) from the patients with a negative test result (shown in Fig. 1).

\section{Discussion}

Our study was conducted as a prospective pilot study to test for plasma EGFR ctDNA in patients with early and locally advanced staged lung adenocarcinoma with biopsy-proven EGFR mutations. These patients underwent initial plasma EGFR ctDNA testing with ARMS PCR followed by radical radiotherapy. The trial was terminated as the investigators felt it was futile to continue due to poor recruitment and low plasma EGFR ctDNA detection rates. The protocol was updated after recruitment was stopped to allow for the retrospective use of NGS 
for better sensitivity [13] and detection of other ctDNA. This improved the overall detection rate of ctDNA from 22.2 to $44.4 \%$.

The ctDNA studies performed in early-stage NSCLC have reported poor detection rates $[7,9,10,14,15]$. Similarly, our detection rate in this early-stage series was poor. We noted that detection rate of plasma EGFR ctDNA was higher in stage III than in stage I/II (60 vs. $0 \%$ ) disease. Although this difference was not statistically significant, ctDNA detection rates have also been noted to be higher in patients with greater tumour burden [7, 16-18]. We also studied the difference in mean GTV and SUVmax values between detectable and undetectable patients as surrogates for tumour load and activity, respectively. The results were, however, not significant. We thus would not recommend its use in early-stage disease outside of clinical trials.

In our study, only 1 patient agreed to post-radiotherapy blood test. This patient had completed 33 fractions of concurrent chemo-radiotherapy and had a subsequent decrease in plasma EGFR ctDNA allele frequency at 1 month post-treatment from 1.7 to $0.8 \%$. Unfortunately, he relapsed radiologically at a distal site shortly after the blood test. From previous surgical series, a detectable plasma EGFR ctDNA after radical surgery suggested the presence of molecular/minimal residual disease (MRD). In these series, the persistence of plasma ctDNA identified patients at high risk of relapse and preceded radiological progression by a median of 70 days to 5.2 months $[7,8,10,19,20]$. This association between post-treatment ctDNA and MRD may apply to radiotherapy patients as well $[8,11]$ and may be useful to complement follow-up radiological scans for diagnosis of relapse.

Univariate analyses of our results showed a negative correlation between a detectable baseline plasma EGFR ctDNA and PFS. This result is consistent with some reports performed in metastatic NSCLC patients [21-25]. Given that ctDNA values are correlated with tumour burden, we speculate that these patients with radiological local disease but detectable baseline plasma EGFR ctDNA may harbour a higher systemic burden and do poorly despite local radical treatment.

Our study is limited due to the small sample size, heterogenous patient cohort and short follow-up period. The interpretation of the NGS technique added later in the protocol could have also resulted in increased false negative results due to ctDNA degradation. However, despite these shortcomings, there are very few studies reporting the use of plasma EGFR ctDNA in non-metastatic NSCLC. Given the poor outcomes of lung cancer patients and difficulties of CT-scan interpretation of post-radical lung radiotherapy, the use of plasma ctDNA could serve as an important biomarker in treatable NSCLC for staging, prognostication, and follow-up.

In conclusion, the clinical applications of ctDNA including plasma EGFR ctDNA in NSCLC patients is evolving. With improved technology and access, the use of non-invasive tests like plasma EGFR ctDNA earlier in the course of disease may be useful to guide future clinical management. The findings of our study suggest that plasma EGFR ctDNA was associated with later-stage disease and may have a negative prognostic value in non-metastatic patients. One patient who had a persistent post-treatment plasma EGFR ctDNA had radiological progression shortly after, possibly indicating some utility for monitoring patient outcomes. These results should be explored in future trials.

\section{Acknowledgements}

The authors wish to thank the team at the Institute of Bioengineering and Nanotechnology, Agency for Science, Technology and Research Singapore for their laboratory contributions.

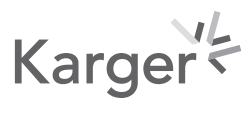




\section{Case Reports in Oncology}

\begin{tabular}{l|l}
\hline Case Rep Oncol 2020;13:896-903 \\
\hline DOI: 10.1159/000508932 & $\begin{array}{l}\text { @ 2020 The Author(s). Published by S. Karger AG, Basel } \\
\text { www.karger.com/cro }\end{array}$ \\
\hline
\end{tabular}

Chia et al.: Plasma EGFR ctDNA Detection Pre-Radical Radiotherapy

\section{Statement of Ethics}

This study protocol was approved by the Ethics Committee of SingHealth Centralised Institutional Review Board. All patients provided written informed consent before recruitment and publication of case. The study was conducted in conformance with the guidelines for Good Clinical Practice and the Declaration of Helsinki as well as the Human Biomedical Research Act.

\section{Conflict of Interest Statement}

Min-Han Tan is the founding CEO and Medical Director of Lucence Diagnostics. All remaining authors have declared no conflicts of interest.

\section{Funding Sources}

This work was supported by the National Medical Research Council, Singapore (grant number NRINSU020100, 2015).

\section{Author Contributions}

Study concept and design: B.S.H.C., W.L.N., M.-H.T. Laboratory contribution: M.-H.T. Statistical analysis: B.S.H.C., S.C. Data interpretation: B.S.H.C., W.L.N., M.-H.T. Drafting and revision of manuscript: B.S.H.C., W.L.N., S.C., K.W.F., M.-H.T. Study supervision: M.-H.T., K.W.F. All authors approved the final revision.

\section{References}

1 Zhang YL, Yuan JQ, Wang KF, Fu XH, Han XR, Threapleton D, et al. The prevalence of EGFR mutation in patients with non-small cell lung cancer: a systematic review and meta-analysis. Oncotarget. 2016;7(48):78985-93.

2 NICE. Plasma EGFR mutation tests for adults with locally advanced or metastatic non-small-cell lung cancer. 2018 [cited 2019 Apr 28]. https://www.nice.org.uk/advice/mib137

3 FDA. US Food and Drug Administration. Summary of safety and effectiveness data: cobas EGFR mutation test v2, 28 September 2016. [cited 2019 Apr 28]. https://www.accessdata.fda.gov/cdrh_docs/pdf15/P150044B. pdf

4 Mok T, Wu YL, Lee JS, Yu CJ, Sriuranpong V, Sandoval-Tan J, et al. Detection and Dynamic Changes of EGFR Mutations from Circulating Tumor DNA as a Predictor of Survival Outcomes in NSCLC Patients Treated with First-line Intercalated Erlotinib and Chemotherapy. Clin Cancer Res. 2015;21(14):3196-203.

5 Oxnard GR, Paweletz CP, Kuang Y, Mach SL, O'Connell A, Messineo MM, et al. Noninvasive Detection of Response and Resistance in EGFR-Mutant Lung Cancer Using Quantitative Next-Generation Genotyping of Cell-Free Plasma DNA. Clin Cancer Res. 2014;20(6):1698-705.

6 Sorensen BS, Wu L, Wei W, Tsai J, Weber B, Nexo E, et al. Monitoring of epidermal growth factor receptor tyrosine kinase inhibitor-sensitizing and resistance mutations in the plasma DNA of patients with advanced non-small cell lung cancer during treatment with erlotinib. Cancer. 2014;120(24):3896-901.

7 Abbosh C, Birkbak NJ, Wilson GA, Jamal-Hanjani M, Constantin T, Salari R, et al. Phylogenetic ctDNA analysis depicts early-stage lung cancer evolution. Nature. 2017;545(7655):446-51.

8 Chaudhuri AA, Chabon JJ, Lovejoy AF, Newman AM, Stehr H, Azad TD, et al. Early detection of molecular residual disease in localized lung cancer by circulating tumor DNA profiling. Cancer Discov. 2017;7:1394-403.

9 Chen K, Zhang J, Guan T, Yang F, Lou F, Chen W, et al. Comparison of plasma to tissue DNA mutations in surgical patients with non-small cell lung cancer. J Thorac Cardiovasc Surg. 2017;154(3):1123-e2.

10 Guo N, Lou F, Ma Y, Li J, Yang B, Chen W, et al. Circulating tumor DNA detection in lung cancer patients before and after surgery. Sci Rep. 2016;6:33519. 
11 Newman AM, Bratman SV, To J, Wynne JF, Eclov NC, Modlin LA, et al. An ultrasensitive method for quantitating circulating tumor DNA with broad patient coverage. Nat Med. 2014;20(5):548-54.

12 Tumor Treatment - Lucence. [cited 2019 Jun 23]. https://www.lucencedx.com/tumor-treatment/

13 Mlika M, Dziri C, Zorgati MM, Ben Khelil M, Mezni F. Liquid biopsy as surrogate to tissue in lung cancer for molecular profiling: a meta-analysis. Curr Respir Med Rev. 2018;14(1):48-60.

14 Cohen JD, Li L, Wang Y, Thoburn C, Afsari B, Danilova L, et al. Detection and localization of surgically resectable cancers with a multi-analyte blood test. Science. 2018;359(6378):926-30.

15 Fiala C, Diamandis EP. Utility of circulating tumor DNA in cancer diagnostics with emphasis on early detection. BMC Med. 2018 Oct 2;16(1):166.

16 Sacher AG, Paweletz C, Dahlberg SE, Alden RS, O'Connell A, Feeney N, et al. Prospective validation of rapid plasma genotyping for the detection of EGFR and kras mutations in advanced lung cancer. JAMA Oncol. 2016; 2(8):1014-22.

17 Seki Y, Fujiwara Y, Kohno T, Yoshida K, Goto Y, Horinouchi H, et al. Circulating cell-free plasma tumour DNA shows a higher incidence of EGFR mutations in patients with extrathoracic disease progression. ESMO Open. 2018;3(2):e000292.

18 Bettegowda C, Sausen M, Leary RJ, Kinde I, Wang Y, Agrawal N, et al. Detection of circulating tumor DNA in early- and late-stage human malignancies. Sci Transl Med. 2014;6(224):224ra24.

19 Liang W, Zhao Y, Huang W, Liang H, Zeng H, He J. Liquid biopsy for early stage lung cancer. J Thorac Dis. 2018; 10(S7):S876-81.

20 Hu W, Yang Y, Zhang L, Yin J, Huang J, Huang L, et al. Post surgery circulating free tumor DNA is a predictive biomarker for relapse of lung cancer. Cancer Med. 2017;6(5):962-74.

21 Phan TT, Tran B-T, Nguyen ST, Ho TT, Nguyen HT, Le VT, et al. EGFR plasma mutation in prediction models for resistance with EGFR TKI and survival of non-small cell lung cancer. Clin Trans Med. 2019;8(1):4.

22 Yanagita M, Redig AJ, Paweletz CP, Dahlberg SE, O'Connell A, Feeney N, et al. A Prospective Evaluation of Circulating Tumor Cells and Cell-Free DNA in EGFR-Mutant Non-Small Cell Lung Cancer Patients Treated with Erlotinib on a Phase II Trial. Clin Cancer Res. 2016;22(24):6010-20.

23 Lee Y, Park S, Kim WS, Lee JC, Jang SJ, Choi J, et al. Correlation between progression-free survival, tumor burden, and circulating tumor DNA in the initial diagnosis of advanced-stage EGFR-mutated non-small cell lung cancer. Thorac Cancer. 2018;9(9):1104-10.

24 Karachaliou N, Mayo-de las Casas C, Queralt C, de Aguirre I, Melloni B, Cardenal F, et al. Association of EGFR L858R Mutation in Circulating Free DNA With Survival in the EURTAC Trial. JAMA Oncol. 2015;1(2):149-57.

25 Kim CG, Shim HS, Hong MH, Cha YJ, Heo SJ, Park HS, et al. Detection of activating and acquired resistant mutation in plasma from EGFR-mutated NSCLC patients by peptide nucleic acid (PNA) clamping-assisted fluorescence melting curve analysis. Oncotarget. 2017;8(39):65111-22. 